Supplement of Atmos. Chem. Phys. Discuss., 15, 31153-31196, 2015

http://www.atmos-chem-phys-discuss.net/15/31153/2015/

doi:10.5194/acpd-15-31153-2015-supplement

(C) Author(s) 2015. CC Attribution 3.0 License.

(c) (i)

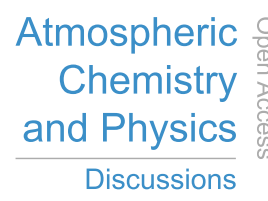

Supplement of

\title{
Role of methyl group number on SOA formation from aromatic hydrocarbons photooxidation under low $\mathrm{NO}_{\boldsymbol{x}}$ conditions
}

L. Li et al.

Correspondence to: D. R. Cocker III (dcocker@engr.ucr.edu)

The copyright of individual parts of the supplement might differ from the CC-BY 3.0 licence. 
Table S1 Aromatic hydrocarbon physical properties and rate constant

\begin{tabular}{ccccc}
\hline Compound & Vapor Pressure $^{\mathrm{a}}$ & Boiling Point $^{\mathrm{b}}$ & $\mathrm{k}_{\mathrm{OH}}{ }^{\mathrm{c}}$ & ${\text { SAPRC- } \mathrm{kOH}^{\mathrm{d}}}^{\mathrm{S}}$ \\
\hline Benzene & 75 & 80 & 0.139 & 0.122 \\
Toluene & 21 & 111 & 0.563 & 0.558 \\
$m$-Xylene & 9 & 139 & 2.31 & 2.31 \\
1,2,4-trimethylbenzene & 2.1 & 170 & 3.25 & 3.25 \\
1,2,4,5-tetramethylbenzene & $5.28 \mathrm{E}-1$ & 193 & 5.55 & 4.10 \\
Pentamethylbenzene & $3.48 \mathrm{E}-02^{*}$ & 232 & 10.3 & 7.63 \\
Hexamethylbenzene & $8.60 \mathrm{E}-04$ & 265 & 11.3 & 11.3 \\
\hline
\end{tabular}

2 Note: a) vapor pressures are referred to Chemispider in unit mmHg at $25^{\circ} \mathrm{C}$; b) boiling points are referred to

3 Chemispider in unit ${ }^{\circ} \mathrm{C}$; c) $\mathrm{OH}$ reaction rate constants are refer to Calvert, et al, 2002; Atkinson and Arey, 2003;

4 Aschmann, et al, 2013 in unit $10^{-11} \mathrm{~cm}^{3}$ molecule ${ }^{-1} \mathrm{~s}^{-1}$ at $25^{\circ} \mathrm{C}$. d) $\mathrm{OH}$ reaction rate constants used in SAPRC-11

5 model in unit $10^{-11} \mathrm{~cm}^{3}$ molecule $\mathrm{e}^{-1} \mathrm{~s}^{-1}$ at $25^{\circ} \mathrm{C}$; *Predicted data from Chemispider.

6 Table S2 Experimental conditions for additional $m$-xylene experiments from Song, et al.

(2005)

\begin{tabular}{|c|c|c|c|c|c|c|}
\hline ID & $\mathrm{HC} / \mathrm{NO}^{\mathrm{a}}$ & $\mathrm{NO}^{\mathrm{b}}$ & $\mathrm{HC}^{\mathrm{b}}$ & $\Delta \mathrm{HC}^{\mathrm{c}}$ & $\mathrm{M}_{0}^{\mathrm{c}}$ & Yield \\
\hline $104 \mathrm{~A}$ & 10.1 & 64.4 & 350 & 328 & 21.7 & 0.07 \\
\hline 104B & 29.3 & 21.4 & 338 & 281 & 20.4 & 0.07 \\
\hline $107 \mathrm{~A}$ & 26.0 & 89.6 & 1254 & 1029 & 146 & 0.14 \\
\hline $129 \mathrm{~A}$ & 15.1 & 45.5 & 371 & 336 & 21.9 & 0.07 \\
\hline $149 \mathrm{~A}$ & 13.3 & 50.2 & 360 & 342 & 52.8 & 0.15 \\
\hline $164 \mathrm{~A}$ & 12.4 & 44.0 & 293 & 271 & 16.8 & 0.06 \\
\hline 164B & 12.2 & 44.1 & 291 & 270 & 14.6 & 0.05 \\
\hline $217 \mathrm{~A}$ & 36.8 & 8.90 & 176 & 155 & 9.80 & 0.06 \\
\hline $217 \mathrm{~B}$ & 35.9 & 8.70 & 168 & 153 & 7.90 & 0.05 \\
\hline $219 \mathrm{~A}$ & 63.7 & 7.00 & 240 & 165 & 9.20 & 0.06 \\
\hline $219 \mathrm{~B}$ & 67.5 & 6.60 & 240 & 166 & 9.30 & 0.06 \\
\hline $288 \mathrm{~A}$ & 63.1 & 7.00 & 238 & 183 & 9.00 & 0.05 \\
\hline $290 \mathrm{~A}$ & 31.1 & 15.3 & 256 & 229 & 9.00 & 0.04 \\
\hline $293 \mathrm{~A}$ & 29.9 & 13.7 & 221 & 189 & 9.20 & 0.05 \\
\hline $368 \mathrm{~A}$ & 17.9 & 21.0 & 203 & 149 & 6.90 & 0.05 \\
\hline $485 \mathrm{~A}$ & 17.5 & 43.3 & 408 & 353 & 37.2 & 0.11 \\
\hline $485 \mathrm{~B}$ & 16.7 & 45.0 & 404 & 349 & 40.4 & 0.12 \\
\hline $488 \mathrm{~A}$ & 15.5 & 46.2 & 386 & 341 & 29.5 & 0.09 \\
\hline $492 \mathrm{~A}$ & 13.6 & 44.3 & 324 & 296 & 29.1 & 0.10 \\
\hline $492 B$ & 13.5 & 44.8 & 325 & 298 & 29.7 & 0.10 \\
\hline $566 \mathrm{~A}$ & 14.0 & 48.3 & 364 & 337 & 48.2 & 0.14 \\
\hline $566 \mathrm{~B}$ & 13.3 & 48.0 & 344 & 318 & 48.4 & 0.15 \\
\hline $758 \mathrm{~A}$ & 47.5 & 11.4 & 292 & 158 & 13.5 & 0.09 \\
\hline $820 \mathrm{~A}$ & 30.2 & 20.7 & 337 & 260 & 17.0 & 0.07 \\
\hline
\end{tabular}

8

9 Note: a) Unit of $\mathrm{HC} / \mathrm{NO}$ are ppbC:ppb; b) Unit of $\mathrm{NO}$ and $\mathrm{HC}$ are $\mathrm{ppb}$; c) Unit of $\Delta \mathrm{HC}$ and $\mathrm{M}_{0}$ are $\mu \mathrm{g} \cdot \mathrm{m}^{-3}$ 
1 Table S3 Average radical concentrations throughout photooxidation

\begin{tabular}{|c|c|c|c|c|c|c|c|c|}
\hline Run ID & $\mathrm{RO}_{2}{ }^{\mathrm{a}}$ & $\mathrm{HO}_{2}{ }^{\mathrm{a}}$ & $\mathrm{OH}^{\mathrm{b}}$ & $\mathrm{HO}_{2} * \mathrm{RO}^{\mathrm{c}}$ & $\mathrm{HO}_{2} / \mathrm{RO}_{2}$ & $\mathrm{NO} / \mathrm{HO}_{2}$ & $\mathrm{OH} / \mathrm{HO}_{2}{ }^{\mathrm{d}}$ & $\mathrm{NO}_{3}{ }^{\mathrm{a}}$ \\
\hline $1236 \mathrm{~A}$ & 12.9 & 23.5 & 4.9 & 530 & 2.7 & $4.9 \mathrm{E}+03$ & $3.3 \mathrm{E}-02$ & 1.8 \\
\hline $1236 \mathrm{~B}$ & 1.30 & 4.4 & 4.7 & 30.4 & 3.9 & $5.9 \mathrm{E}+05$ & 3.6E-01 & 8.3 \\
\hline $1237 \mathrm{~A}$ & 15.2 & 20.8 & 7.6 & 488 & 2.3 & $3.1 \mathrm{E}+04$ & $1.4 \mathrm{E}-01$ & 1.9 \\
\hline 1237B & 13.7 & 20.7 & 5.6 & 416 & 2.3 & $2.3 \mathrm{E}+02$ & $1.2 \mathrm{E}-02$ & 0.9 \\
\hline $1223 \mathrm{~A}$ & 10.5 & 24.0 & 4.7 & 376 & 2.7 & $1.5 \mathrm{E}+04$ & $6.2 \mathrm{E}-02$ & 1.7 \\
\hline $1618 \mathrm{~A}$ & 9.50 & 107.2 & 5.5 & 1194 & 13.5 & $3.1 \mathrm{E}+01$ & $1.1 \mathrm{E}-03$ & 6.2 \\
\hline $1223 B$ & 10.0 & 16.4 & 7.0 & 508 & 3.2 & $1.7 \mathrm{E}+08$ & $4.3 \mathrm{E}+01$ & 5.6 \\
\hline $1101 \mathrm{~A}$ & 15.7 & 19.3 & 6.8 & 335 & 1.4 & $5.8 \mathrm{E}+01$ & $7.8 \mathrm{E}-03$ & 4.0 \\
\hline 1101B & 16.3 & 18.5 & 5.0 & 320 & 1.2 & $8.9 \mathrm{E}+00$ & $3.8 \mathrm{E}-03$ & 1.4 \\
\hline $1102 \mathrm{~A}$ & 12.9 & 17.3 & 10 & 313 & 1.7 & $1.6 \mathrm{E}+04$ & $1.2 \mathrm{E}-01$ & 16.2 \\
\hline 1102B & 13.8 & 17.5 & 8.7 & 328 & 1.6 & $1.0 \mathrm{E}+03$ & $2.8 \mathrm{E}-02$ & 10.5 \\
\hline $1106 \mathrm{~A}$ & 7.30 & 12.3 & 10 & 118 & 1.9 & $8.1 \mathrm{E}+02$ & 3.9E-02 & 16.3 \\
\hline 1106B & 9.30 & 14.5 & 7.9 & 144 & 1.6 & $1.5 \mathrm{E}+01$ & 8.2E-03 & 6.0 \\
\hline $1468 \mathrm{~A}$ & 23.8 & 24.5 & 3.9 & 716 & 1.3 & $6.3 \mathrm{E}+01$ & $3.2 \mathrm{E}-03$ & 11.8 \\
\hline 1468B & 26.4 & 26.3 & 4.1 & 740 & 1.0 & $4.6 \mathrm{E}+01$ & $3.1 \mathrm{E}-03$ & 4.8 \\
\hline $1193 \mathrm{~A}$ & 11.0 & 12.3 & 3.1 & 185 & 1.3 & $1.5 \mathrm{E}+06$ & $1.8 \mathrm{E}+00$ & 14.0 \\
\hline 1193B & 9.00 & 11.6 & 2.8 & 141 & 1.4 & $3.2 \mathrm{E}+05$ & 4.2E-01 & 18.7 \\
\hline $1191 \mathrm{~A}$ & 19.1 & 15.8 & 5.8 & 449 & 1.2 & $4.5 \mathrm{E}+05$ & $3.4 \mathrm{E}-01$ & 32.0 \\
\hline 1191B & 10.8 & 12.4 & 2.4 & 190 & 1.5 & $3.8 \mathrm{E}+04$ & $5.1 \mathrm{E}-02$ & 18.5 \\
\hline $1516 \mathrm{~A}$ & 18.6 & 23.1 & 3.1 & 465 & 1.3 & $1.5 \mathrm{E}+01$ & $2.0 \mathrm{E}-03$ & 2.4 \\
\hline $1950 \mathrm{~A}$ & 11.7 & 20.6 & 4.5 & 267 & 1.8 & $7.2 \mathrm{E}+01$ & 4.3E-03 & 38.4 \\
\hline 1950B & 13.3 & 22.0 & 4.6 & 326 & 1.7 & $4.6 \mathrm{E}+01$ & 3.7E-03 & 37.1 \\
\hline $1117 \mathrm{~A}$ & 13.1 & 15.8 & 1.7 & 220 & 1.3 & $1.2 \mathrm{E}+01$ & $1.5 \mathrm{E}-03$ & 7.3 \\
\hline 1117B & 9.80 & 14.6 & 2.5 & 172 & 1.6 & $1.9 \mathrm{E}+02$ & 4.7E-03 & 34.9 \\
\hline $1119 \mathrm{~A}$ & 12.5 & 18.8 & 5.3 & 300 & 1.7 & $2.7 \mathrm{E}+03$ & $1.7 \mathrm{E}-02$ & 89.0 \\
\hline 1119B & 12.2 & 17.3 & 4.0 & 296 & 1.9 & $2.9 \mathrm{E}+03$ & $1.7 \mathrm{E}-02$ & 68.6 \\
\hline $1123 \mathrm{~A}$ & 15.9 & 15.1 & 1.7 & 274 & 1.1 & $6.8 \mathrm{E}+01$ & 2.2E-03 & 2.9 \\
\hline 1123B & 15.8 & 18.6 & 2.5 & 321 & 1.2 & $7.7 \mathrm{E}+01$ & $3.0 \mathrm{E}-03$ & 23.2 \\
\hline $1126 \mathrm{~A}$ & 17.3 & 17.6 & 1.7 & 324 & 1.1 & $1.7 \mathrm{E}+01$ & $1.5 \mathrm{E}-03$ & 4.3 \\
\hline $1126 \mathrm{~B}$ & 30.0 & 24.8 & 7.5 & 841 & 1.0 & $3.2 \mathrm{E}+01$ & $3.8 \mathrm{E}-03$ & 33.7 \\
\hline 1129B & 11.2 & 15.4 & 4.3 & 199 & 1.6 & $5.6 \mathrm{E}+01$ & 4.9E-03 & 24.0 \\
\hline $1531 \mathrm{~A}$ & N/A & N/A & N/A & N/A & N/A & N/A & N/A & $\mathrm{N} / \mathrm{A}$ \\
\hline $1603 \mathrm{~A}$ & 40.9 & 21.8 & 1.6 & 971 & 0.7 & $2.3 \mathrm{E}+01$ & $9.5 \mathrm{E}-04$ & 0.6 \\
\hline 1603B & 38.9 & 20.4 & 1.6 & 925 & 0.8 & $7.7 \mathrm{E}+01$ & $1.3 \mathrm{E}-03$ & 0.6 \\
\hline $2085 \mathrm{~A}$ & 31.5 & 28.3 & 1.2 & 971.5 & 1.1 & $3.2 \mathrm{E}+01$ & 6.7E-04 & 2.2 \\
\hline $2085 B$ & 27.4 & 22.7 & 0.6 & 642.2 & 1.0 & $3.7 \mathrm{E}+00$ & $3.2 \mathrm{E}-04$ & 0.5 \\
\hline
\end{tabular}




\begin{tabular}{lcccccccc}
$1488 \mathrm{~A}$ & N/A & N/A & N/A & N/A & N/A & N/A & N/A & N/A \\
$1521 \mathrm{~A}$ & 24.7 & 23.5 & 0.8 & 621 & 1.0 & $4.7 \mathrm{E}+01$ & $5.4 \mathrm{E}-04$ & 1.2 \\
$1627 \mathrm{~A}$ & 27.8 & 23.0 & 1.0 & 702 & 1.0 & $5.7 \mathrm{E}+01$ & $6.9 \mathrm{E}-04$ & 1.0 \\
$1627 \mathrm{~B}$ & 15.5 & 22.2 & 2.1 & 439 & 1.6 & $4.0 \mathrm{E}+03$ & $4.9 \mathrm{E}-03$ & 47.5 \\
$1557 \mathrm{~A}$ & 10.1 & 16.7 & 0.2 & 171 & 1.7 & $1.6 \mathrm{E}+00$ & $9.3 \mathrm{E}-05$ & 0.5 \\
$2083 \mathrm{~A}$ & 13.5 & 15.6 & 0.9 & 280 & 1.3 & $1.6 \mathrm{E}+06$ & $6.0 \mathrm{E}-01$ & 1.1 \\
$2083 \mathrm{~B}$ & 10.0 & 17.0 & 1.2 & 213 & 1.8 & $4.0 \mathrm{E}+02$ & $2.0 \mathrm{E}-03$ & 13.8 \\
\hline
\end{tabular}

1 Note: average radical concentrations are calculated by dividing time integrated radical parameters with photooxidation time;

2 average radical concentration throughout photooxidation a) in $10^{6}$ molecules $\cdot \mathrm{cm}^{-3}$; b) in $10^{8}$ molecules $\cdot \mathrm{cm}^{-3}$; c) in

$310^{16}$ molecules $\cdot \mathrm{cm}^{-3} ; \mathrm{d}$ ) average radical ratio throughout photooxidation in $10^{3}$

4 Table S4 Correlation between SOA yields and average radical concentrations

\begin{tabular}{lcccccccc}
\hline & $\mathrm{RO}_{2}$ & $\mathrm{HO}_{2}$ & $\mathrm{OH}$ & $\mathrm{HO}_{2} * \mathrm{RO}_{2}$ & $\mathrm{HO}_{2} / \mathrm{RO}_{2}$ & $\mathrm{NO} / \mathrm{HO}_{2}$ & $\mathrm{OH} / \mathrm{HO}_{2}$ & $\mathrm{NO}_{3}$ \\
\hline Yield & -0.243 & 0.169 & 0.459 & 0.067 & 0.261 & 0.294 & 0.292 & -0.237 \\
p-value $^{\mathrm{a}}$ & 0.125 & 0.292 & 0.003 & 0.678 & 0.099 & 0.062 & 0.064 & 0.136 \\
\hline
\end{tabular}

5 Note: a) P-values range from 0 to 1, 0-reject null hypothesis and 1 accept null hypothesis. Alpha ( $\alpha$ ) level used is 0.05 . If the

6 p-value of a test statistic is less than alpha, the null hypothesis is rejected

7 Table S5 Correlation among SOA density, volatility (VFR), SOA chemical composition and

8 methyl group number

\begin{tabular}{lcccccccc}
\hline & Density & $\mathrm{VFR}_{\text {end }}{ }^{\mathrm{a}}$ & $\mathrm{f}_{44}$ & $\mathrm{f}_{43}$ & $\mathrm{H} / \mathrm{C}$ & $\mathrm{O} / \mathrm{C}$ & $\mathrm{OS}_{\mathrm{c}}$ & Methyl $^{\mathrm{c}}$ \\
\hline Density & - & 0.715 & 0.790 & -0.839 & -0.756 & $\mathbf{0 . 8 7 3}$ & 0.834 & -0.943 \\
p-value $^{\mathrm{b}}$ & - & 0.071 & 0.034 & 0.018 & 0.049 & 0.01 & 0.02 & 0.001 \\
VFR $_{\text {end }^{\mathrm{a}}}$ & 0.715 & - & 0.768 & -0.896 & -0.905 & $\mathbf{0 . 9 3 7}$ & 0.932 & -0.838 \\
p-value $^{\mathrm{b}}$ & 0.071 & - & 0.044 & 0.006 & 0.005 & 0.002 & 0.002 & 0.0019
\end{tabular}

Note: a) $\mathrm{VFR}_{\text {end }}$ volume remaining fraction at the end of photooxidation; b) P-values range from 0 to 1,0 -reject null hypothesis

10 and 1 accept null hypothesis. Alpha $(\alpha)$ level used is 0.05 . If the p-value of a test statistic is less than alpha, the null hypothesis is

11 rejected; c) Methyl group number is used for statistical analysis

12 Table S6 Vapor pressure predication of selected benzene photooxidation products

\begin{tabular}{lcc}
\hline Formula & Reaction pathway & Predicted $\log \mathrm{P}_{\text {vap }}{ }^{a}$ \\
\hline $\mathrm{C}_{6} \mathrm{H}_{6} \mathrm{O}_{5}$ & $\mathrm{~S}_{1}$, Bicyclic peroxide & $-3.83 \mathrm{E}+00$ \\
$\mathrm{C}_{6} \mathrm{H}_{6} \mathrm{O}_{8}$ & $\mathrm{~S}_{1}$, Bicyclic peroxide & $-6.39 \mathrm{E}+00$
\end{tabular}



$\mathrm{C}_{8} \mathrm{H}_{10} \mathrm{O}_{10}$
$\mathrm{S}_{3}$, Oligomerization, c-2-1
$-1.13 \mathrm{E}+01$
$\mathrm{C}_{8} \mathrm{H}_{10} \mathrm{O}_{9}$
$\mathrm{S}_{3}$, Oligomerization, c-1-1
$-7.47 \mathrm{E}+00$

$\mathrm{C}_{6} \mathrm{H}_{8} \mathrm{O}_{6} \quad \mathrm{~S}_{3}$, Oligomerization, c-2-1, with glyoxal

$-6.92 \mathrm{E}+00$

Note : a) Prediction is based on Pankow and Asher 2008, $\log \mathrm{P}_{\text {vap }}$ is in the unit of $\log (\mathrm{atm})$
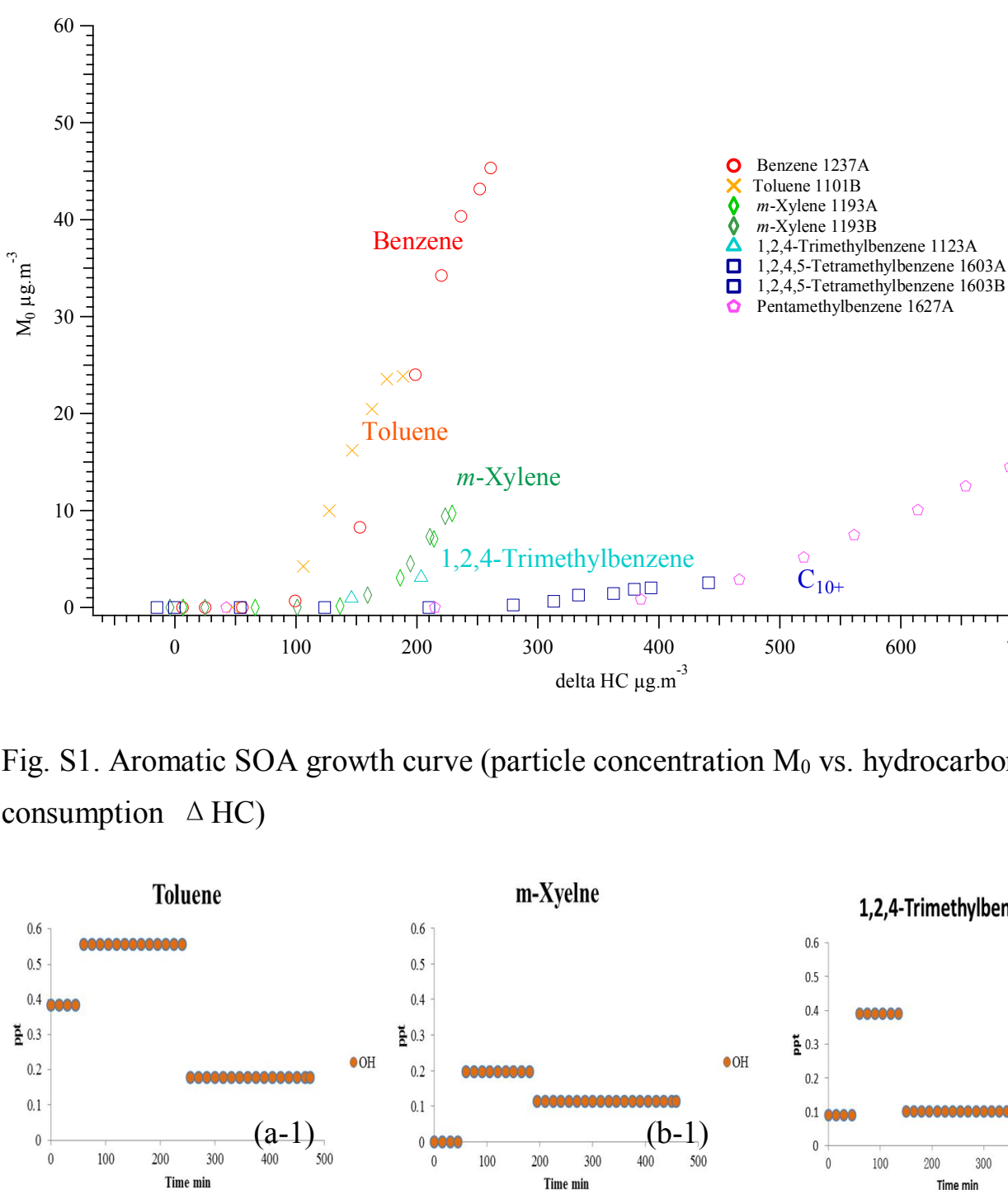

2

Toluene

$m$-Xylene

$\circ \quad \infty$

1,2,4-Trimethylbenzene $\square \quad \mathrm{C}_{10+}$

200

$300 \quad 400 \quad 500$

$600 \quad 700 \quad 800$

delta $\mathrm{HC} \mu \mathrm{g} \cdot \mathrm{m}^{-3}$

3 Fig. S1. Aromatic SOA growth curve (particle concentration $\mathrm{M}_{0}$ vs. hydrocarbon

4 consumption $\triangle \mathrm{HC}$ )

5

6

8

9

10

11

12

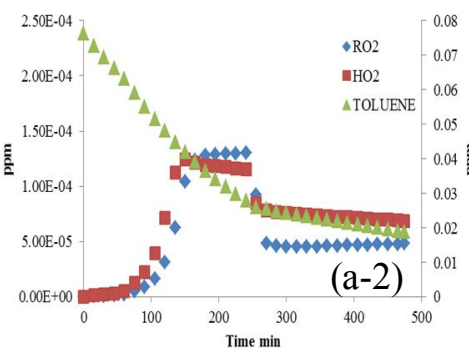

m-Xyelne

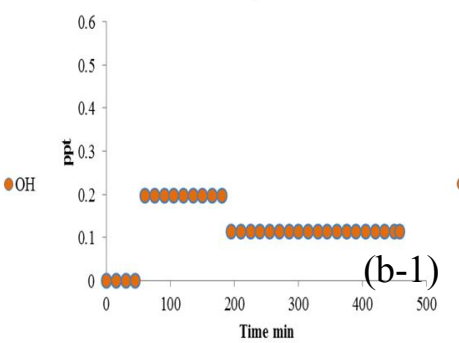

1,2,4-Trimethylbenzene

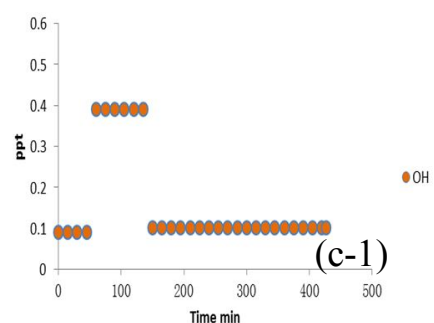

13

Fig. S2. Time series radical profile during photooxidation (a) toluene 1102B; (b) $m$-xylene

14 1193A; (c) 1,2,4-trimethylbenzene 1119B) 

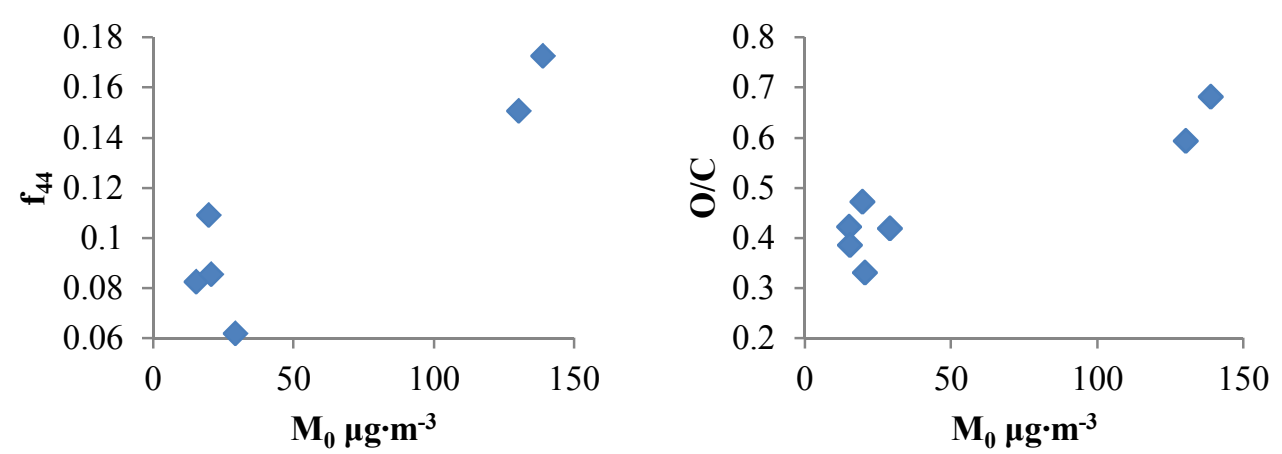

1

2 Fig.S3. Relationship between $\mathrm{f}_{44}, \mathrm{O} / \mathrm{C}$ and mass loading

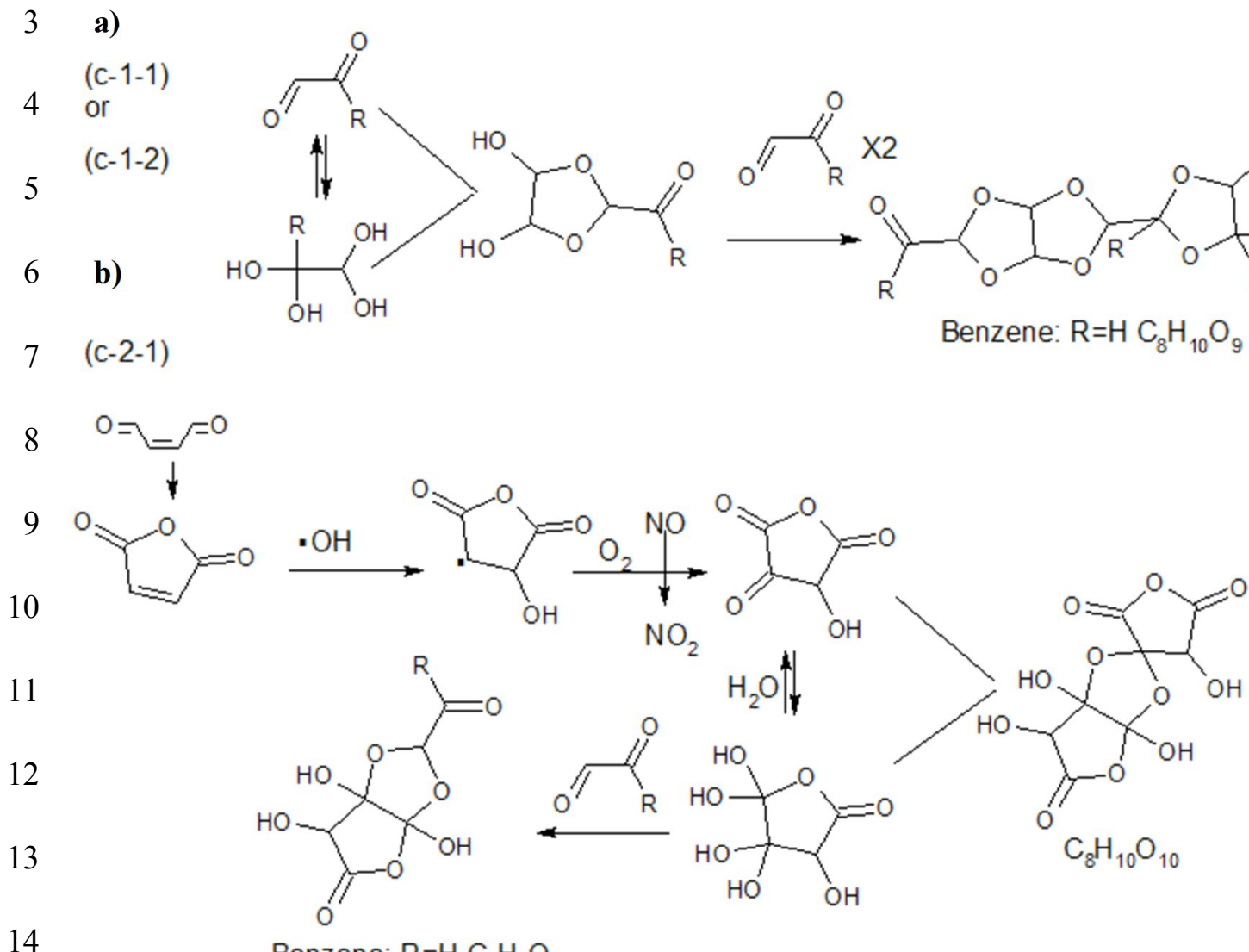

(c-2-2)

16

17

$0=\stackrel{\mathrm{H}_{3} \mathrm{C}}{=}=0$

18

19
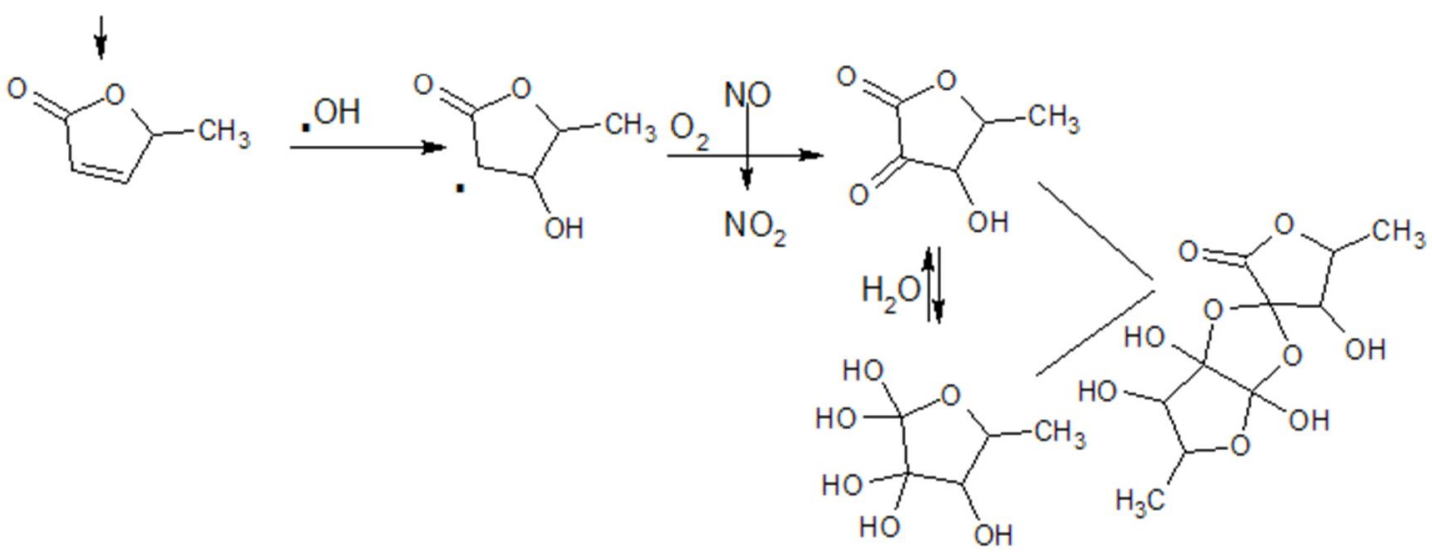
1 Fig. S4. Potential oligomerization pathways during aromatic hydrocarbon photooxidation a)

2 from saturated 1,2-dicarbonyls to oligomers (adopted from Kalberer, et al, 2004); b) from

3 unsaturated 1,4-dicarbonys to oligomers (c-1-1, c-1-2, c-2-1 and c-2-2 are pathways

4 mentioned in Fig. 7.).

5

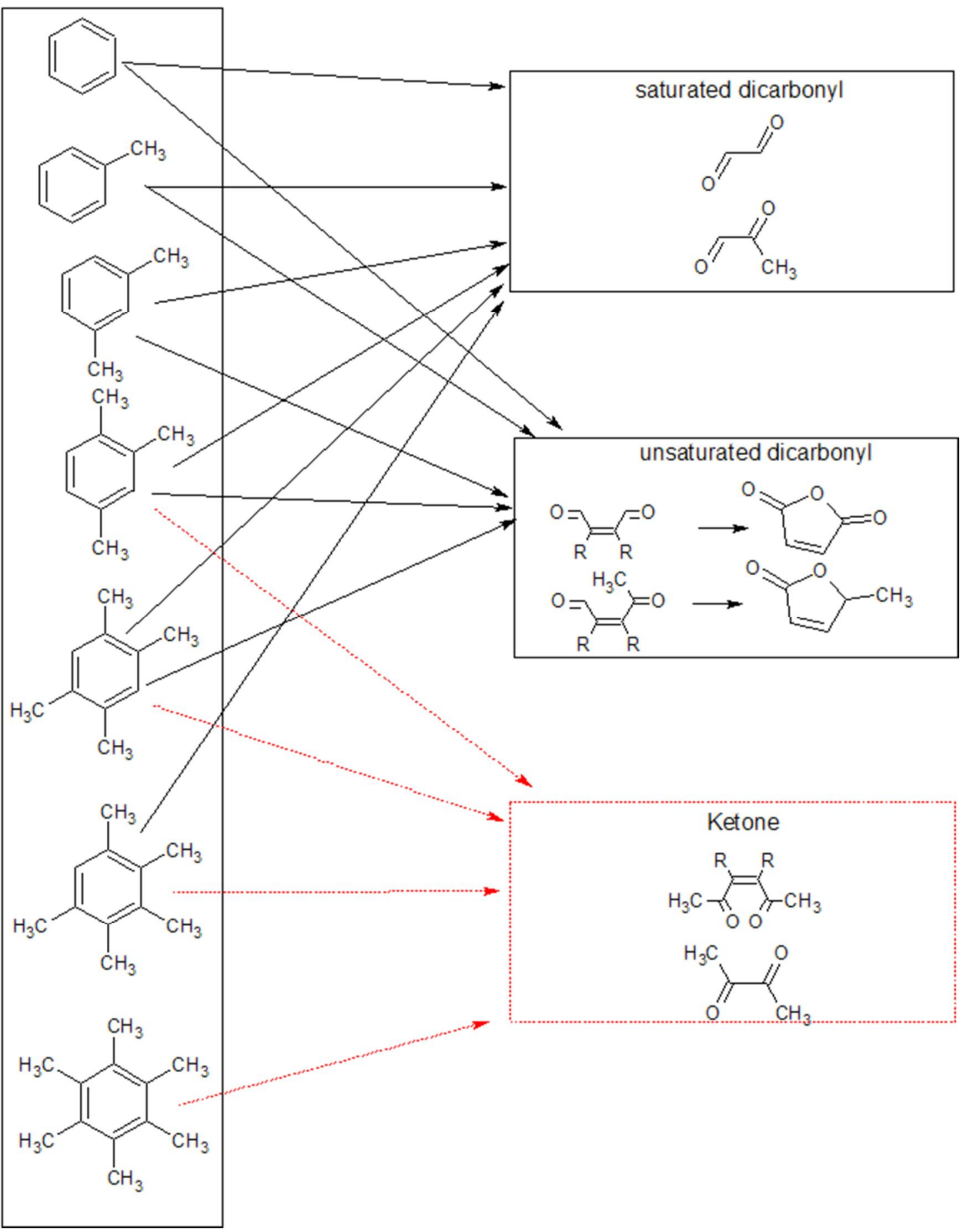


1 Fig S5. Potential ring opening products of aromatic hydrocarbons during photooxidation $(\mathrm{OH}$

2 attach to ring carbon not occupied by a methyl group is the only pathway considered in

3 pentamethylbenzene photoxidation)

4 